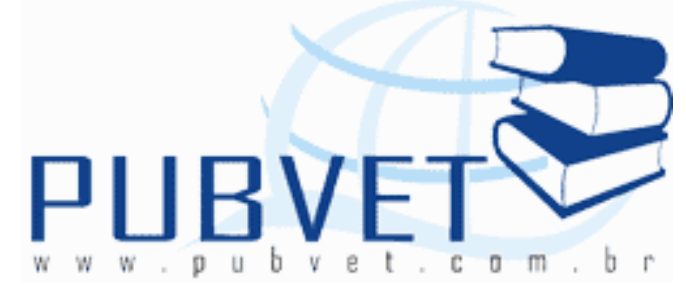

PUBVET, Publicações em Medicina Veterinária e Zootecnia.

\title{
Variabilidade genética de características produtivas e de qualidade de ovos de matrizes de codornas de corte
}

Raquel Pillon Della-Flora ${ }^{1}$, Ariane Gotuzzo ${ }^{1}$, Jerusa Martins Germano ${ }^{2}$, Carolina Bavaresco2, Viviane Lacerda², Nelson José Laurino Dionello ${ }^{3}$

${ }_{1}^{1}$ Mestrandas em Zootecnia/Bolsista do CnPq. Departamento de Zootecnia. FAEM/UFPel .Pelotas, RS.

${ }^{2}$ Alunos do curso de Zootecnia. FAEM/UFPel. Pelotas, RS.

${ }_{3}^{3}$ Prof.Dr. e Bolsista de produtividade do CNPq. Departamento de Zootecnia.

FAEM/UFPel.Pelotas, RS.

\section{Resumo}

O trabalho estudou características de produção de ovos e de qualidade interna e externa de ovos de uma linhagem de codornas de corte, selecionada após oito gerações no Laboratório de Ensino e Experimentação Animal prof. Dr. Renato Rodrigues Peixoto (LEEZO) do Departamento de Zootecnia da Universidade Federal de Pelotas. Foram avaliadas 250 codornas de corte na fase de postura dos 42 aos 126 dias em duas gerações sucessivas. O modelo animal levou em consideração os efeitos fixos de geração e os efeitos aleatórios aditivos e residuais, em uma análise unicaracterística. As características avaliadas foram peso da ave, produção e massa de ovos, consumo alimentar e conversão alimentar por massa e dúzia de ovos; peso do ovo, casca, clara e gema e cor da gema como características internas dos ovos e largura e comprimento dos ovos, gravidade específica e altura de albúmen, 
como características externas dos ovos, respectivamente. Os resultados mostraram elevadas herdabilidades para peso da ave $(0,49)$, produção de ovos $(0,37)$, peso da gema $(0,35)$, clara $(0,53)$ e ovos $(0,59)$, e comprimento $(0,32)$. A variabilidade encontrada nas características produtivas e de qualidade dos ovos sugere a possibilidade de ganhos genéticos nessas características pela seleção fenotípica, principalmente para peso do ovo e da clara e produção de ovos.

Palavras-chave: herdabilidades, peso de clara, gema e ovos, produção de ovos, variâncias

\title{
Genetic variability for eggs production and quality traits in meat quail
}

\begin{abstract}
The work studied traits for egg production and internal and external quality traits of a line of meat quail, selected after eight generations in Professor Renato Rodrigues Peixoto Animal Research and Teaching Laboratory (LEEZO) of Department of Zootecnia of Federal University of Pelotas. Were evaluated 250 meat quail eggs productions of 42 to 126 days in two successive generations. The animal model took into consideration the fixed effects for generation and random additives and residual effects in a unitraits analysis. The traits evaluated were body weight, egg production and egg mass, food consumption and feed conversion ratio by mass and dozen eggs; weight yolk, shell, albumen and eggs and yolk color as internal traits of eggs and width and length of eggs, specific gravity and height of albumen, as external traits of eggs, respectively. The results showed high heritability to body weight (0.49), egg production (0.37), yolk weight (0.35), albumen weight (0.53), egg weight (0.59) and length eggs (0.32). The variability found in productive traits and quality of the eggs suggests the possibility of genetic gains on these traits by phenotypic selection, mainly to albumen and egg weight and egg production.
\end{abstract}

Keywords: albumen, egg and yolk weight, egg production, heritability, variances 


\section{INTRODUÇÃO}

A produção de codornas é um segmento importante na indústria avícola brasileira apresentando aumento significativo nos anos recentes, sendo que entre os anos de 1990 e 2007 apresentou um aumento de $208 \%$ (Hidalgo et al., 2011). Os principais fatores que contribuíram para isto são a carne, a qual apresenta um sabor exótico, o ovo, o qual é semelhante ao ovo de aves e é considerado afrodisíaco, o rápido crescimento das codornas, a precocidade sexual com início de produção aos 35 dias, o baixo investimento inicial, entre outros (Murakami \& Ariki, 1998). O desenvolvimento do material genético em codornas assemelha-se ao realizado em aves, tanto frangos de corte ou poedeiras, com o desenvolvimento de linhagens selecionadas, onde visa-se obter a expressão do efeito genético aditivo e o possível uso da heterose como forma de recuperar este efeito quando ocorra queda do mesmo por causa da consangüinidade (Martins, 2002). Em codornas de corte é importante a verificação da produção de ovos das matrizes, pois isto vai ser determinante da condição reprodutiva que a linhagem vai apresentar refletindo-se no nascimento de codorninhas para a próxima geração. Diversas características são importantes na avaliação interna e externa dos ovos de codornas de corte em fase de postura, além é claro da própria produção de ovos das matrizes. Assim o desenvolvimento de uma linhagem implica na estimação de parâmetros genéticos com a finalidade de tomada de decisão baseada nas avaliações de características produtivas realizadas. O objetivo deste trabalho foi estimar as herdabilidades para características na fase de postura até 126 dias, considerado período parcial, em uma linhagem de codornas de corte selecionada após oito gerações. 


\section{MATERIAL E MÉTODOS}

O trabalho foi desenvolvido no Laboratório de Ensino e Experimentação Zootécnica Professor Dr. Renato Rodrigues Peixoto do DZ/FAEM/UFPel, onde avaliaram-se as produções de 250 codornas de corte de duas gerações sucessivas e nas idades de 42-126 dias, o que pode ser considerado um período parcial de produção, sendo as análises qualitativas realizadas no Laboratório de Ovos. Utilizou-se o método de máxima verossimilhança restrita (REML) através do Programa Wombat, em uma análise uni característica que proporcionou a obtenção entre outros resultados das variâncias genéticas aditivas, ambientais e fenotípicas. O modelo estatístico utilizado na análise das características considerou o efeito fixo de geração e os efeitos aleatórios genéticos aditivos diretos e de resíduos. Esse modelo pode ser representado, na forma matricial, por $Y=X \beta+Z a+e$, em que: $Y$ representa o vetor de observações; $\beta$ a matriz de incidência dos efeitos fixos; $X$ o vetor de efeitos fixos; a a matriz de incidência dos efeitos aleatórios genéticos aditivos diretos; $Z$ o vetor de efeitos aleatórios genéticos aditivos diretos; e e o vetor de resíduos aleatórios. As pressuposições assumidas para os efeitos aleatórios foram as seguintes:

$$
\left(\begin{array}{l}
Y \\
\mathrm{a} \\
e
\end{array}\right] \sim \mathrm{NMV}\left\{\left[\begin{array}{l}
\beta \\
\phi \\
\phi
\end{array}\right],\left[\begin{array}{ccc}
G Z^{\prime}+R & Z G & R \\
G Z^{\prime} & G & \phi \\
R & \phi & R
\end{array}\right]\right\} \text {, em que, } \mathrm{G}=\mathrm{A} \sigma^{2}{ }_{\mathrm{a}} \text { e } \mathrm{R}=\mathrm{I} \quad \sigma_{\mathrm{e}}^{2}
$$

sendo G a matriz de (co)variâncias genéticas aditivas, A a matriz de numeradores do coeficiente de parentesco e $\sigma^{2}$ a variância genética aditiva; $R$ a matriz de variância residual, sendo I a matriz identidade e $\sigma^{2}$ e variância residual.

As características analisadas foram peso das aves (PA); produção de ovos totais (PROD); consumo de ração (CR) e massa de ovos (MO) diários; 
conversão alimentar por quilo ( $\mathrm{CA} / \mathrm{MO})$ e dúzia de ovos $(\mathrm{CA} / \mathrm{dz})$ produzidos; gravidade específica (GE); pesos da casca (Pcasca), gema (Pgema), ovo (povo) e clara (Pclara); largura (larg) e comprimento (comp); altura de albúmen (AA) e cor da gema (corgema), respectivamente. A avaliação das características de qualidade dos ovos foi realizada ao final do período e durante três dias, os ovos foram pesados individualmente em uma balança digital com sensibilidade de $0,5 \mathrm{~g}$ e o comprimento e largura foram obtidos através de um paquímetro digital. A gravidade específica foi determinada pelo método de flutuação salina, conforme metodologia descrita por Hamiltom (1982). Os ovos, em número de três, foram acondicionados em cesto de fundo perfurado e imersos em recipientes contendo concentração salina crescente, variaram de $1,054 \mathrm{~g} / \mathrm{cm}^{3}$ a $1,94 \mathrm{~g} / \mathrm{cm}^{3}$ com intervalo de $0,004 \mathrm{~g} / \mathrm{cm}^{3}$, totalizando 11 soluções. A cada 70-80 ovos a solução salina do recipiente era calibrada por um densímetro. A altura do albúmen foi verificada com uma régua específica. Após a separação do albúmen da gema do ovo esta foi pesada em uma balança digital com sensibilidade de $0,5 \mathrm{~g}$ e da mesma forma posteriormente, 0 albúmen. $O$ peso das cascas de ovos foi aferido em balança com precisão de centigramas.

\section{RESULTADOS E DISCUSSÃO}

Para as características consideradas produtivas (Tabela 1), o valor de herdabilidade obtido para a característica produção de ovos foi muito interessante, sendo comparável ao valor obtido por Minvielle et al. (1997) de 0,39, para o período de 91 dias de idade, sendo esperado que esta característica pudesse ser usada na seleção, com expectativa de ganho genético. Este resultado apresenta-se superior ao obtido por Dionello et al. (2011) que com a mesma linhagem selecionada na terceira geração não encontraram esta expressiva variabilidade. Sabidamente no caso de aves de postura, esta característica não apresenta grande variabilidade sendo difícil 
DELLA-FLORA, R.P. et al. Variabilidade genética de características produtivas e de qualidade de ovos de matrizes de codornas de corte. PUBVET, Londrina, V. 6, N. 33, Ed. 220, Art. 1463, 2012.

sua seleção e dependendo em grande parte da pressão de seleção utilizada para melhorá-la ao longo das gerações. Igualmente o peso da ave apresentouse com alta herdabilidade $(0,49 \pm 0,15)$ mas não seria uma característica importante para a produção de ovos, entretanto deve estar relacionada com o peso de ovos, principalmente nesta linhagem pesada. Para a massa de ovos os valores foram comparáveis aos obtidos por Dionello et al. (2011) quando trabalharam com a mesma linhagem na terceira geração. As demais características apresentaram herdabilidades que seriam classificadas como de médias-baixas. A característica de conversão alimentar por dúzia de ovos foi a que apresentou menor herdabilidade e ao mesmo tempo um elevadíssimo erro padrão. Para as características de qualidade interna dos ovos (Tabela 2), peso da clara e da gema, seriam classificadas como de médias-altas herdabilidades. Essas características, além do peso do ovo, mostraram expressiva variabilidade genética pressupondo-se que poderiam ser usadas na seleção, com expectativa de ganho genético. A variabilidade do peso do ovo, especificamente no caso de ovos grandes demais, caso típico de linhagens pesadas, poderia causar maior pressão nas bandejas inferiores, durante a coleta, com consequente maior índice de quebra (Teixeira et al., 2012), sendo que este tamanho excessivo também pode ser notado ao impedir o fechamento da embalagem de 30 ovinhos. Futuramente, por esta razão a ideia de uniformidade dos ovos pode ser um padrão exigido pelo consumidor. Já a variabilidade existente para característica peso da gema $(0,35 \pm 0,15)$ pode ser a responsável pela variação no tempo de incubação e nascimento das codorninhas que pode ocorrer entre 17 e 21 dias, segundo Teixeira et al. (2012). Ainda segundo estes autores, o atraso ou a extensão nesse tempo de incubação podem reduzir a eclodibilidade, quando o manejo estabelece a retirada num tempo pré-fixado. A extensão do período, com vistas a melhorar a qualidade de codornas no nascimento ou alojamento, ganhou maior atenção com a determinação dos efeitos negativos nos parâmetros de desempenho pós-nascimento, decorrentes do atraso no fornecimento de ração e (ou) água. Considerando que o peso ao nascimento está correlacionado com os 
componentes dos ovos, a uniformização dos ovos poderia ser praticada para homogeneizar o nascimento, o que viria a reduzir o estresse das aves nessa fase inicial, gerando melhor desempenho das codornas. De modo geral a estimativa obtida para peso do ovo $(0,59 \pm 0,15)$ está bem acima do valor obtido por Baungartner (1994) de 0,35 e por Saatci et al. (2006) de 0,25. Também estaria dentro do intervalo de valores reportados por Minvielle (1998) e Mielenz et al. (2004), que oscilou entre 0,35 e 0,66 e acima do valor obtido por Özdemir \& Aksit (2004), que encontraram resultados de herdabilidade de 0,48 ao utilizarem dados de peso de ovo medido em várias idades, todos autores trabalhando com codornas de postura. A cor da gema não apresentou variabilidade genética e ao mesmo tempo um alto erro padrão, enquanto, peso da casca apresentou baixa herdabilidade. Para as características de qualidade externa dos ovos (Tabela 3), para altura de albúmen o valor de herdabilidade encontrado está abaixo do obtido por Özdemir \& Aksit (2004) e Georg et al. (2009) que encontraram herdabilidades em torno de 0,30. Para largura e comprimento de ovos os valores obtidos foram comparáveis aos obtidos por Teixeira et al. (2012). De modo geral para todas as características estudadas, as herdabilidades mostraram elevados valores dos erros padrão o que pode ser explicado pelo baixo número de aves, embora para características como estas se tornasse difícil se avaliar um número muito grande de aves devido a demora no período de coleta para obtenção das características e também sabendo-se que ocorre queda na produção com o aumento na idade, já reportada em outros trabalhos. 
DELLA-FLORA, R.P. et al. Variabilidade genética de características produtivas e de qualidade de ovos de matrizes de codornas de corte. PUBVET, Londrina, V. 6, N. 33, Ed. 220, Art. 1463, 2012.

Tabela 1 - Variâncias genéticas aditivas, residuais e herdabilidades ( \pm erro padrão) de características de produção de ovos de matrizes de codornas de corte

\begin{tabular}{lllllll}
\hline & PA & PROD & CR & MO & CA/MO & CA/dz \\
\hline$\sigma^{2}{ }_{a}$ & 785,87 & 89,57 & 1,51 & 1,13 & 0,35 & 0,01 \\
$\sigma^{2}{ }_{e}$ & 812,04 & 152,35 & 6,54 & 5,28 & 2,42 & 0,07 \\
$h^{2}$ & $0,49 \pm 0,15$ & $0,37 \pm 0,16$ & $0,19 \pm 0,12$ & $0,18 \pm 0,14$ & $0,13 \pm 0,16$ & $0,01 \pm 0,12$
\end{tabular}

$\sigma^{2}{ }_{a}$ - variância genética aditiva; $\sigma^{2}{ }_{e}-$ variância residual; $h^{2}-$ herdabilidade

Tabela 2 - Variâncias genéticas aditivas, residuais e herdabilidades ( \pm erro padrão) de características de qualidade internas de ovos de matrizes de codornas de corte

\begin{tabular}{llllll}
\hline & Povo & Pcasca & Pclara & Pgema & corgema \\
\hline$\sigma^{2}{ }_{a}$ & 0,896 & 0,003 & 0,404 & 0,078 & 0,0001 \\
$\sigma^{2}{ }_{e}$ & 0,619 & 0,023 & 0,356 & 0,148 & 0,196 \\
$h^{2}$ & $0,59 \pm 0,15$ & $0,11 \pm 0,14$ & $0,53 \pm 0,15$ & $0,35 \pm 0,15$ & $0,00 \pm 0,11$
\end{tabular}

$\sigma^{2}{ }_{a}$ - variância genética aditiva; $\sigma^{2}{ }_{e}-$ variância residual; $h^{2}-$ herdabilidade

Tabela 3 - Variâncias genéticas aditivas, residuais e herdabilidades ( \pm erro padrão) de características de qualidade externas de ovos de matrizes de codornas de corte

\begin{tabular}{lllll}
\hline \multicolumn{1}{c}{ Larg } & Comp & GE & AA \\
\hline$\sigma^{2}{ }_{a}$ & 0,0020 & 0,007 & 0,00005 & 0,069 \\
$\sigma^{2}{ }_{e}$ & 0,0067 & 0,015 & 0,0001 & 0,368 \\
$h^{2}$ & $0,23 \pm 0,14$ & $0,32 \pm 0,15$ & $0,27 \pm 0,14$ & $0,16 \pm 0,14$
\end{tabular}

$\sigma^{2}{ }_{a}$ - variância genética aditiva; $\sigma^{2}{ }^{2}$-variância residual; $h^{2}$ - herdabilidade 
DELLA-FLORA, R.P. et al. Variabilidade genética de características produtivas e de qualidade de ovos de matrizes de codornas de corte. PUBVET, Londrina, V. 6, N. 33, Ed. 220, Art. 1463, 2012.

\section{CONCLUSÕES}

A variabilidade encontrada nas características produtivas e de qualidade dos ovos, nas matrizes avaliadas para produção de codorna de corte sugerem a possibilidade de ganhos genéticos nessas características pela seleção fenotípica, especialmente para peso do ovo e da clara e produção de ovos.

\section{AGRADECIMENTOS}

Ao Conselho Nacional de Desenvolvimento Científico e Tecnológico (CNPq) e ao Programa de Pós Graduação em Zootecnia da Universidade Federal de Pelotas, pelo suporte financeiro.

\section{REFERÊNCIAS BIBLIOGRÁFICAS}

BAUNGARTNER, J. Japanese quail production breeding and genetics. World's Poultry Science, v.50, n.3, p.228-235, 1994.

DIONELLO, N.J.L.; ROLL, V.F.B.; XAVIER, E.G. et al. Produção em número, peso e massa de ovos em codornas de corte até 406 dias de idade. PUBVET, Londrina, V. 5, N. 27, Ed. 174, Art. 1172, 2011.

GEORG, P.C.; PAIVA, E.; CONTI, A.C.M. et al. Interação genótipo X ambiente em codornas de postura alimentadas com rações com dois níveis de energia metabolizável. R. Bras. Zootec. [online]. vol.38, n.9, pp. 1706-1711, 2009..

HAMILTOM, R.M.G. Methods and factors that affect the measurement off egg shell quality. Poultry Science, v.61, p. 2002-2039, 1982.

HIDALGO, A.M.; MARTINS, E.N.; SANTOS, A.L. et al. Genetic characterization of egg weight, egg production and age at first egg in quails. R. Bras. Zootec., v.40, n.1, p.95-99, 2011.

MARTINS, E.N. Perspectivas do melhoramento genético de codornas no Brasil. In: SIMPÓSIO INTERNACIONAL DE COTURNICULTURA, 1., 2002, Lavras. Anais... Lavras: UFLA, 2002. p.109112. 
MIELENZ, N.; NOOR, R.R.; SCHUELLER, L. Estimation of additive and non-additive genetic variances of body weight, egg weight and egg production in quail, using animal models. In: WORLD'S POULTRY CONGRESS, 22., 2004, Istanbul. Proceedings... Istanbul: Turkey. World's Poultry Congress \& Exibition, [2004]. (CD-ROM).

MINVIELLE, F. Genetics and breeding of japanese quail for production around the world. In: ASIAN PACIFIC POULTRY CONGRESS, 6., 1998, Nagoia. Proceedings... Nagoia: Japan Poultry Science Association, 1998. p.122-127.

MINVIELLE, F.; MONVOISIN, J.L.; COSTA J. et al. Quail lines selected on egg number either on pure line or on crossbred performance. In: INTERNATIONAL SYMPOSIUM, 12., 1997, Pruhonice. Proceedings... Pruhonice, 1997.

MURAKAMI, A.E.; ARIKI, J. Produção de codornas japonesas. Jaboticabal: Funep, 1998. 79p.

ÖZDEMIR, D.; AKSIT, M. Estimations of genetic parameters of some egg quality characteristics of Japanese quails (Coturnix coturnix japonica) at different ages. In: WORLD'S POULTRY CONGRESS, 22., 2004, Istanbul. Proceedings... Istanbul: World's Poultry Congress \& Exibition, [2004]. (CD-ROM).

SAATCI, M.; OMED, H.; AP DEWI, I. Genetic parameters from univariate and bivariate analyses of egg and weight traits in japanese quail. Poultry Science, v.85, 2006. p.185-190.

TEIXEIRA, B.B.; TEIXEIRA, R.B.; SILVA, R.P. et al. Estimação dos componentes de variância para as características de produção e de qualidade de ovos em matrizes de codorna de corte. Ciência Rural, v.42, n.4, p. 713-717. 2012. 